\title{
¿Response of Northern Hemisphere Weather and Climate to Arctic Sea Ice Decline: Resolution Independence in Polar Amplification Model Intercomparison Project (PAMIP) Simulations
}

\author{
Jan Streffing, ${ }^{\mathrm{a}, \mathrm{b}}$ Tido Semmler, ${ }^{\mathrm{a}}$ Lorenzo ZAMPIERI ${ }^{\mathrm{a}}$ And ThOMAS Jung ${ }^{\mathrm{a}, \mathrm{c}}$ \\ ${ }^{\mathrm{a}}$ Alfred Wegener Institute Helmholtz Centre for Polar and Marine Research, Bremerhaven, Germany \\ ${ }^{\mathrm{b}}$ Jacobs University Bremen, Bremen, Germany \\ ${ }^{\mathrm{c}}$ University of Bremen, Bremen, Germany
}

(Manuscript received 30 December 2019, in final form 26 July 2021)

\begin{abstract}
The impact of Arctic sea ice decline on the weather and climate in midlatitudes is still much debated, with observations suggesting a strong link and models a much weaker link. In this study, we use the atmospheric model OpenIFS in a set of model experiments following the protocol outlined in the Polar Amplification Model Intercomparison Project (PAMIP) to investigate whether the simulated atmospheric response to future changes in Arctic sea ice fundamentally depends on model resolution. More specifically, we increase the horizontal resolution of the model from 125 to $39 \mathrm{~km}$ with 91 vertical levels; in a second step, resolution is further increased to $16 \mathrm{~km}$ with 137 levels in the vertical. The model does produce a response to sea ice decline with a weaker midlatitude Atlantic jet and increased blocking in the high-latitude Atlantic, but no sensitivity to resolution can be detected with 100 members. Furthermore, we find that the ensemble convergence toward the mean is not impacted by the model resolutions considered here.
\end{abstract}

KEYWORDS: Atmosphere; Arctic; Extratropics; Northern Hemisphere; Teleconnections; Jets

\section{Introduction}

Extreme events such as cold-air outbreaks, long-lasting atmospheric winter blocking, and heavy snow conditions have often been connected with the strongly declining Arctic sea ice in recent decades (Vihma 2014; Overland et al. 2011; Cohen et al. 2018b), not only in the scientific literature but also in the media. The idea of this linkage is that a reduced meridional temperature gradient between the Arctic and northern midlatitudes decelerates the mean westerly flow through the thermal wind relationship and therefore modifies the characteristics of midlatitude winter climate and weather (Holton 2004). This has been investigated both from observation or reanalysis data and from numerical model simulations.

On the observational side, most studies find correlations between Arctic amplification (AA) and shifting midlatitude temperature, precipitation, and wind speed extremes. However, their driving mechanisms have not been fully quantified (Cohen et al. 2014, 2019). The major limitation of observational studies is that of the relatively short observational period, compounded by the large Arctic internal variability (AIV) and intermittency of the teleconnections (Overland et al. 2016). Another complication is the fact that the AA-induced changes on the midlatitude weather patterns have been described as being in a tug of war with those from the tropical high troposphere, another region where

๖ Denotes content that is immediately available upon publication as open access.

Corresponding author: Jan Streffing, jan.streffing@awi.de accelerated changes due to global warming occur (Shaw et al. 2016), thus impeding causal research.

Because of these difficulties, numerous modeling efforts to dissect the AA into its various components have been made. In particular, one major branch is seeking to solve the question of whether and how the reduction of the sea ice cover impacts the midlatitudes. Even though this branch eliminates interference from many other aspects of AA, the results have nevertheless been contradictory, with many groups finding teleconnections (Chen et al. 2016a; Mori et al. 2019; Nakamura et al. 2016; Luo et al. 2016; Kug et al. 2015; Screen et al. 2015), while others conclude that no statistically significant effect exists in their models (Sun et al. 2016; Meleshko et al. 2016; Chen et al. 2016b). The causality of the observational co-occurrence of low sea ice concentration (SIC) leading to colder Eurasian winters was questioned by Blackport et al. (2019), who, through physical considerations and lead-lag analysis, identify the atmospheric anomalies outside of AA as the driver of colder Eurasian winters.

A major issue in the modeling studies has been many different model experiment designs. This issue has compromised the comparability between the previous studies, which may have been part of the reason for the contradicting results. Therefore, a coordinated suite of experiments, the Polar Amplification Model Intercomparison Project (PAMIP) within the framework of CMIP6, has been designed to eliminate the interference of different forcing and experiment designs. The simulations presented herein follow the specifications outlined in

This article is licensed under a Creative Commons Attribution 4.0 license (http://creativecommons.org/ licenses/by/4.0/).

DOI: 10.1175/JCLI-D-19-1005.1 
a) T159

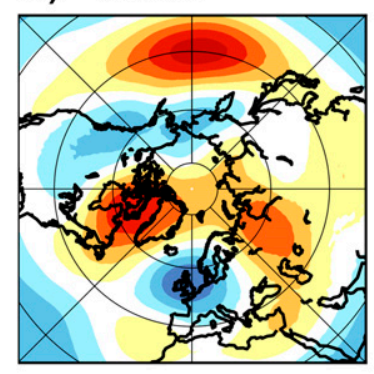

b) $\mathrm{T} 511$

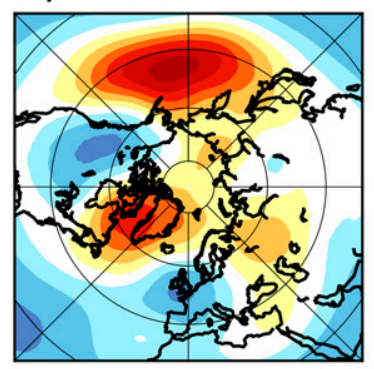

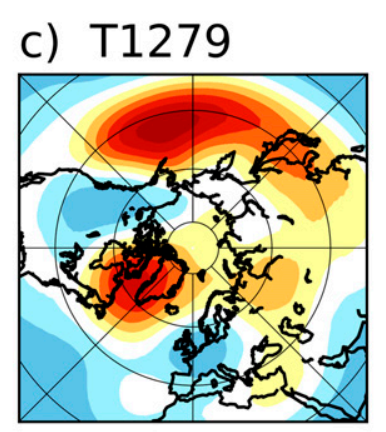

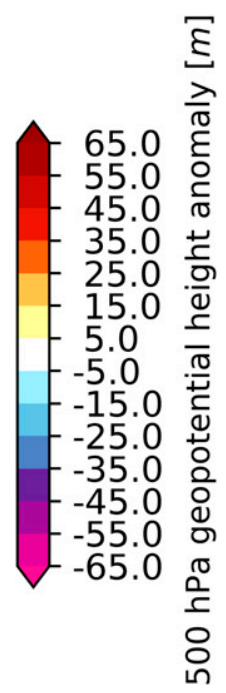

FIG. 1. The 500-hPa geopotential height bias (in m) PD vs ERA5 for PAMIP experiments at three different resolutions: (a) $\mathrm{T}_{\mathrm{L}} 159$, (b) $\mathrm{T}_{\mathrm{L}} 511$, and (c) $\mathrm{T}_{\mathrm{L}} 1279$.

the PAMIP model experiment description (Smith et al. 2019) and are specified further in section $2 \mathrm{~b}$. The results of our study can thus be compared with results from other climate models. The uncertainty of the results is reduced to the model formulation and not anymore dependent on different experiment design.

However, due to the inherent strong natural variability in the mid- and high-latitude atmosphere and due to interaction of the midlatitudes with subtropical and tropical regions the signal-to-noise ratio is very low. Model results have been contradictory, especially with respect to extreme events. The nature of the causal pathway, and in the case of some studies even the existence of the links, has been questioned frequently, leading to a polarized discussion in the scientific community (Cohen et al. 2019; Blackport and Screen 2020b). In the wider atmospheric modeling community there is a continuous trend toward higher horizontal resolutions, which has led to improvements in the simulation of orographic drag (Pithan et al. 2016), atmospheric blocking (Schiemann et al. 2017; Davini et al. 2017), storm tracks (Willison et al. 2015), precipitation (van Haren et al. 2015), and mean surface level pressure (Hertwig et al. 2015). Specifically, as recently shown by Baker et al. (2019), an atmospheric general circulation model (AGCM) at high resolution of $25 \mathrm{~km}$ can potentially react to global warming with stronger shifts of the jet stream and storm track activity than it can at a lower resolution of $135 \mathrm{~km}$. We also know that modest gains in the seasonal forecast skill for sea level pressure, tropical velocity potential anomalies, and representation of blocking events were made by increasing the horizontal resolution of the IFS atmospheric climate model (Jung et al. 2012; Davini and D'Andrea 2016). An improved blocking climatology, especially over the North Atlantic, may be obtained due to a better representation of the eddy-mean flow interaction (Scaife et al. 2019). A high horizontal resolution helps to represent small-scale atmospheric eddies. Additionally, a better representation of topography and large-scale circulation is beneficial to the representation of wind and precipitation extremes (Iles et al. 2019). In the following we explore if a very high horizontal resolution can provide a different answer to the question how Arctic sea ice decline may influence the winter climate of the northern midlatitudes. We will make use of the exceptional computational efficiency of the employed AGCM, OpenIFS (Stevens et al. 2019). Our low-resolution setup will be comparable to recent works in the field at $1.125^{\circ}$, with higher-resolution runs at $0.352^{\circ}$ and $0.1406^{\circ}$, equivalent to about 125,39 , and $16 \mathrm{~km}$ respectively. All setups feature a high ceiling top at $0.01 \mathrm{hPa}$ and ensemble sizes that follow the PAMIP experiment protocol. We will investigate how a marked resolution increase can influence the atmospheric response to Arctic sea ice decline.

\section{Setup}

\section{a. The OpenIFS atmospheric model}

Experiments within this study were conducted with cycle 40r1 (cy40r1) of the OpenIFS model developed at the European Centre for Medium-Range Weather Forecasts (ECMWF). OpenIFS contains the numerical core and parameterization package of the Integrated Forecast System (IFS), and it is capable of forward model runs only, because the 4D-var data assimilation component of the IFS has been removed from OpenIFS (ECMWF 2014a,b,c). Additionally included components are the land surface model HTESSEL 
a) PD SIC

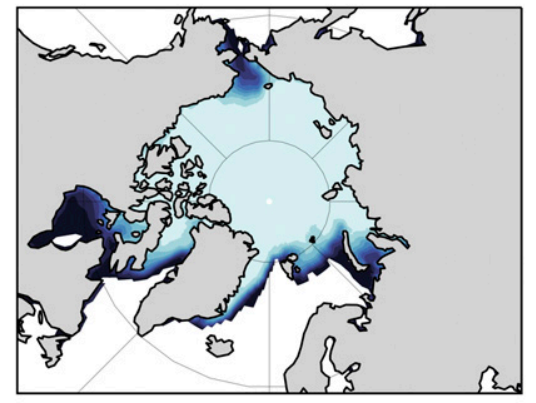

b) $\mathrm{FU} \mathrm{SIC}$

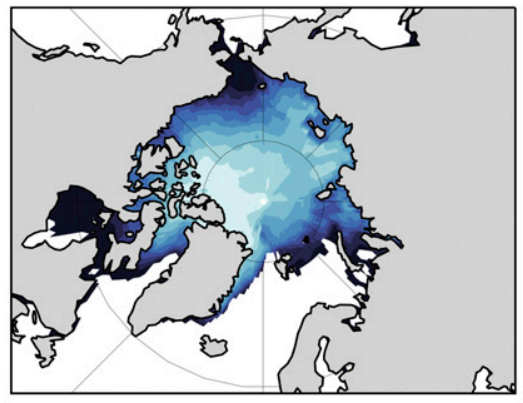

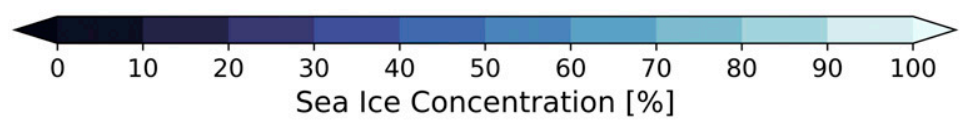

c) Net heat flux

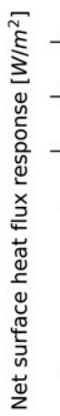

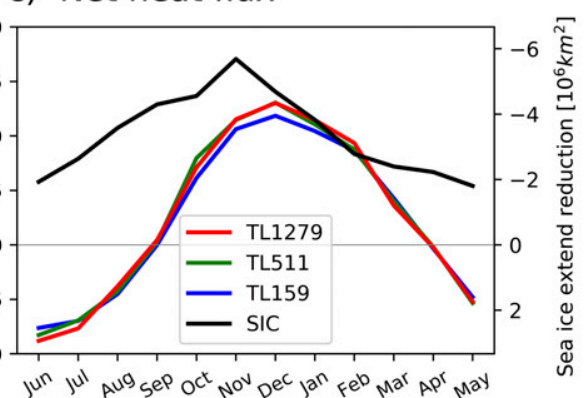

d) $2 \mathrm{~m}$ air temperature

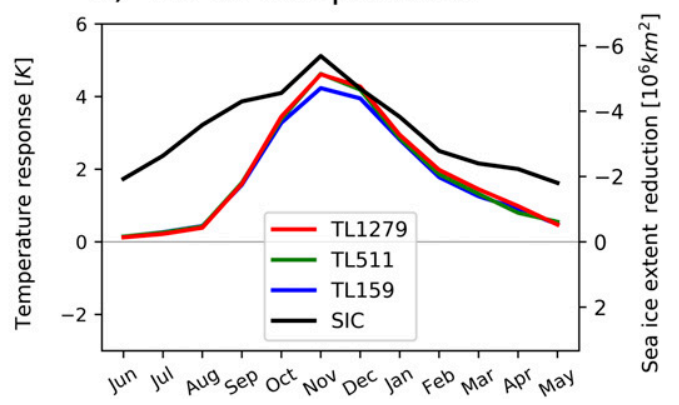

FIG. 2. Sea ice concentration (in \%) for December: (a) present-day (PD) and (b) future forcing (FU). (c) Sea ice extent reduction (in $10^{6} \mathrm{~km}^{2}$ ) from forcing and local response of the net surface heat flux (in $\mathrm{W} \mathrm{m}^{-2}$ ) under a mask of mean March sea ice extent from HadISST 1990-2010. (d) As in (c), but with the 2-m air temperature response (in $\mathrm{K}$ ).

and the wave model WAM, both of which are integrated as modules within OpenIFS. The OpenIFS was developed to give the weather prediction and climate modeling communities easier access to one of the world-leading atmospheric models. OpenIFS makes use of a semi-Lagrangian advection scheme (Staniforth and Côté 1990; Ritchie et al. 1995), determining the origin point of each flow parcel at every time step. The model can thereby skirt the Courant-FriedrichsLewy convergence condition and integrate fewer, longer time steps than models of the same resolution with Eulerian advection, allowing us to efficiently run large ensemble experiments at high resolutions.

TABLE 1. Ensemble sizes of equal predictive power in the midlatitudes $\left(30^{\circ}-70^{\circ} \mathrm{N}\right)$ with regard to four key parameters.

\begin{tabular}{lccc}
\hline \hline & $n_{\mathrm{T}_{\mathrm{L}} 1279}$ & $n_{\mathrm{T}_{\mathrm{L}} 511}$ & $n_{\mathrm{T}_{\mathrm{L}} 159}$ \\
\hline T2M & 100 & 107 & 134 \\
MSLP & 100 & 96 & 110 \\
z500 & 100 & 72 & 82 \\
u500 & 100 & 80 & 95 \\
\hline
\end{tabular}

To gauge the impact of the model resolution on the influence of Arctic sea ice decline, we ran ensemble simulations with three different setups: a low-resolution $\left(\mathrm{T}_{\mathrm{L}} 159\right.$ with 91 horizontal layers), a medium-resolution $\left(\mathrm{T}_{\mathrm{L}} 511\right.$ with 91 horizontal layers), and a high-resolution $\left(\mathrm{T}_{\mathrm{L}} 1279\right.$ with 137 horizontal layers) version. These spherical harmonic truncation numbers are equivalent to $1.125^{\circ} \times 1.125^{\circ}, 0.352^{\circ} \times 0.352^{\circ}$, and $0.1406^{\circ} \times 0.1406^{\circ}$, which amounts to about 125,39 and $16 \mathrm{~km}$ horizontal resolution respectively. The model grids are all of the reduced Gaussian type and, as indicated by the letters $\mathrm{T}_{\mathrm{L}}$, use a linear truncation, meaning the shortest spherical harmonic wavelength is represented by two points in grid point space. The $\mathrm{T}_{\mathrm{L}} 1279$ runs are set up with a higher vertical resolution as this reflects a well-tuned setup, which was used for the operational ECMWF IFS global weather forecasts from November 2013 until May 2015 Bauer and Richardson; Haiden et al. 2015.

\section{b. Experimental setup}

Out of the 24 different experiments in PAMIP only two were chosen for the purpose of this study, one employing presentday SST and SIC data (1.1 pdSST-pdSIC) and one using 


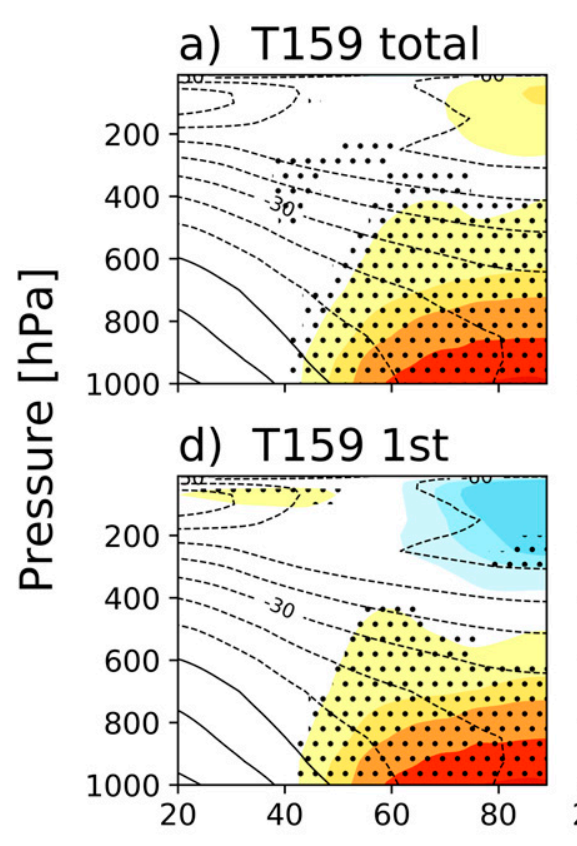

b) T511

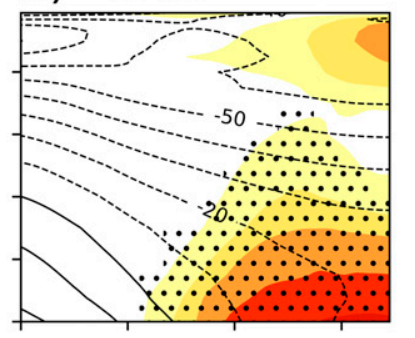

e) T159 2nd

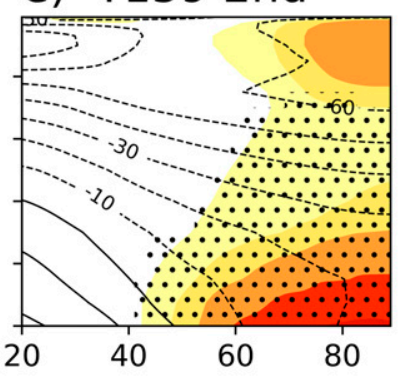

C) $\mathrm{T} 1279$
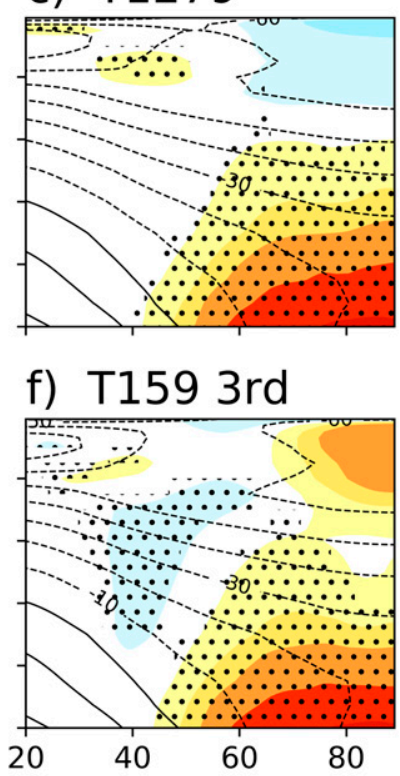

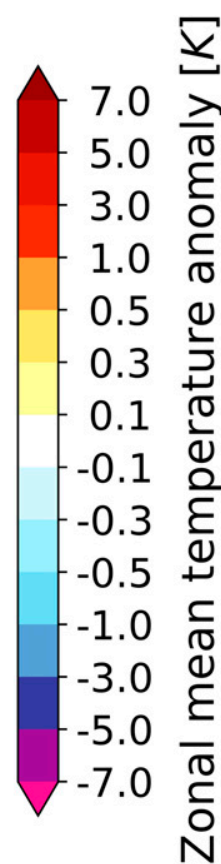

\section{Latitude $\left[{ }^{\circ} \mathrm{N}\right]$}

FIG. 3. Difference in DJFM zonally averaged mean air temperature (in K) between PAMIP ensembles forced with present-day and future Arctic sea ice concentrations: (a) $T_{L} 159$, (b) $T_{L} 511$, and (c) $T_{L} 1279$. (d)-(f) The $T_{L} 159$ ensemble is split into three equal sized sections. Temperatures for the ensemble with future forcing are indicated as contour lines (in ${ }^{\circ} \mathrm{C}$ ). Dots indicate the $95 \%$ bootstrapping confidence interval. Ensemble size is 300 for (a) and 100 for (b)-(f) as described in section 3.

present-day SST and future SIC (1.6 pdSST-futArcSIC). In the following, these experiments will be referred to as PD (present-day experiment) and FU (future experiment). According to the PAMIP protocol, both experiments are tier 1 atmospheric time slices, and they differ only in the prescribed sea ice concentration. PD is forced by climatological monthly mean SST and SIC for the present day, while FU is forced with identical monthly mean SST but with future SIC estimated from model projections. The sea ice temperature is not part of the forcing fields and is instead calculated by the land surface model integrated into the atmospheric GCM, while the sea ice thickness is permanently set to $1.5 \mathrm{~m}$. The boundary conditions for PD were generated as monthly means from the 1979-2008 HadISST dataset. The projections for future SIC are based on a time and ensemble mean that takes into account the first 30 years with $2.0^{\circ} \mathrm{C}$ average global warming from CMIP5 RCP8.5 model runs performed with 31 different models. The process of obtaining the forcing files is described in detail in the appendix of Smith et al. (2019), and forcing fields were collected from the input4MIPs data server (https://esgfnode.1lnl.gov/search/input4mips/, last access: 6 November 2019). (Figure 2 shows the spatial sea ice distribution as well as the monthly evolution of the sea ice extent.) Both timeslice experiments consist of at least 100 individual ensemble members for each of the three employed resolutions and were started from perturbed initial conditions on 1 April
2000. The experiments were run for 14 months, of which only the last 12 are subject to analysis, with the first two months being excluded to allow for model spinup. Following McKenna et al. (2018) we enlarged the ensembles of the $\mathrm{T}_{\mathrm{L}} 159$ experiments with an additional 200 members to test for the robustness of the results obtained by using the ensemble size recommended by PAMIP (i.e., 100 members). Using larger ensembles for T511 and T1279, while in principle desirable, is prohibitively costly, as the required core hours per simulation year increase with the resolutions from 105 to 15000 to 120000 .

\section{Results}

In presenting our model results, we will first follow the proposed causal linkage chains from local to remote changes based on the differential forcing and determine how resolution impacts the outcomes. To quantify the statistical significance of the simulated anomalies we use the bootstrap method by Efron and Tibshirani (1986). In particular, we calculate the point-wise equal-tail bootstrap $P$ value to reject the equality of means with $95 \%$ significance, as detailed in MacKinnon (2009). In section 4 we identify the characteristic signal-to-noise ratio of key variables for local and remote effects and determine how rapidly the ensembles at different resolutions converge toward the ensemble mean. 


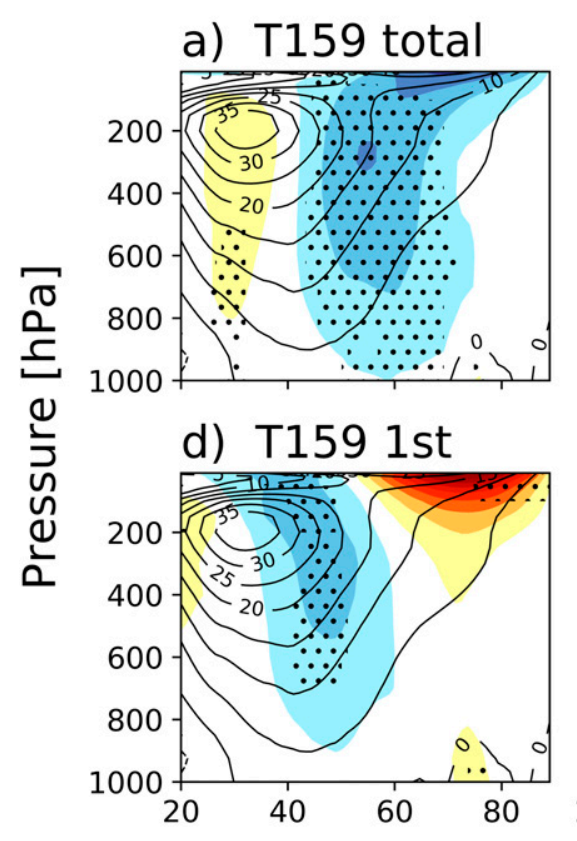

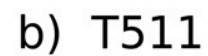
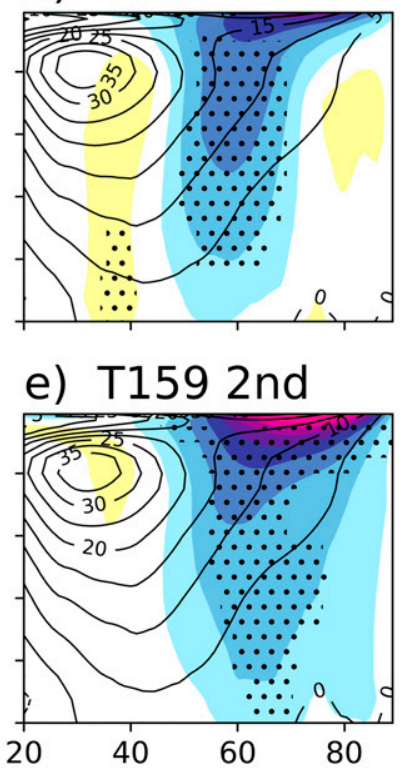

Latitude $\left[{ }^{\circ} \mathrm{N}\right]$ c) $\mathrm{T} 1279$

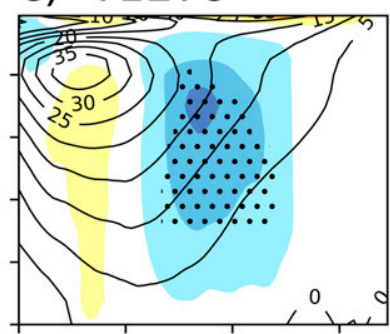

f) $\mathrm{T159} 3 \mathrm{rd}$

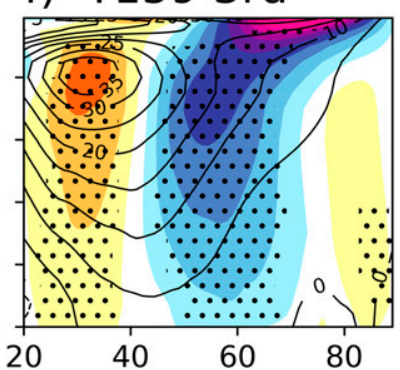

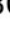

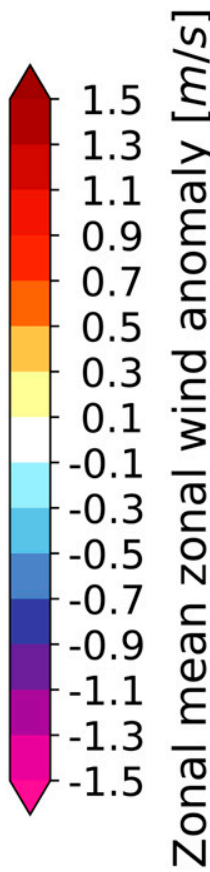

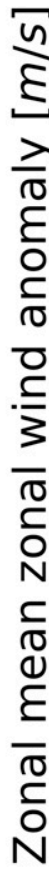

FIG. 4. As in Fig. 3, but for zonally averaged mean zonal wind (in $\mathrm{m} \mathrm{s}^{-1}$ ).

\section{a. Model evaluation}

To put the responses to sea ice decline into perspective, we succinctly show the 500 -hPa geopotential height bias of OpenIFS with present-day forcing compared to ERA5 reanalysis data. We selected the mean of the period from 1990 to 2010 from the reanalysis data, since our simulations were made according to the PAMIP protocol with year 2000 greenhouse gas forcing.

In Fig. 1 we depict the bias of the $500-\mathrm{hPa}$ geopotential height with regard to ERA5. The general pattern of overestimation of the geopotential height over the midlatitude Pacific, Greenland, and the Urals as well as the underestimation in western Europe and eastern North America is common to all three ensembles. Differences exist in strength of for example the Europe-Ural dipole and the strength and positioning of bias maxima in North America and over the Pacific. The 2-m air temperature bias over land (not shown) resembles that shown in Roberts et al. (2018) for IFS 43r1, a close successor of the model used here. Overall our model is slightly colder, likely due to applying the fixes to the supercooled cloud liquid water parameterization developed by (Forbes et al. 2016). This reduced positive radiation bias at high latitudes, leading to an overall colder climate. Over the oceans the near-surface temperature follows closely the differences between the applied HadISST forcing and ERA5.

\section{b. Near-surface response to sea ice forcing}

The forcing response chain starts with a local increase of the sensible, longwave, and latent heat fluxes, due to the reduced extent of the insulating sea ice cover during the cold season (Fig. 2c). This heat flux then affects a local increase in the near-surface air temperature (Fig. 2d). From September until March the delayed and reduced freezing of the Arctic Ocean results in increased upward longwave and turbulent sensible heat fluxes throughout the basin. Net surface heat flux response and the resulting 2-m air temperature changes are shown as monthly means in Fig. 2. The sea ice forcing field provided by PAMIP has a resolution of $1^{\circ}$, independent of our model resolution. Thus the two higher-resolution simulations are forced with a sea ice field smoother than possible at native model resolution. This experiment design limitation could lead to alterations of the local ocean to air heat fluxes that cannot be checked within the PAMIP experiment design. We show that with undersampling of the $1^{\circ}$ forcing field at low resolution and oversampling at high resolution, the local near-surface temperature response differs by less than $0.2 \mathrm{~K}$ between experiments. The wintertime integrated local temperature and heat flux response are only marginally weaker at the lowest resolution, and we would expect this difference to propagate forward through the response chains.

\section{Signal-to-noise ratio}

Before we disseminate the midlatitude response of the model to the applied forcing we will undertake a short excursion on the signal-to-noise ration of the modeled response and quantify potential sampling errors made in this study. To this end we calculate specific root-mean-square errors (RMSE) for 
a) T159 total

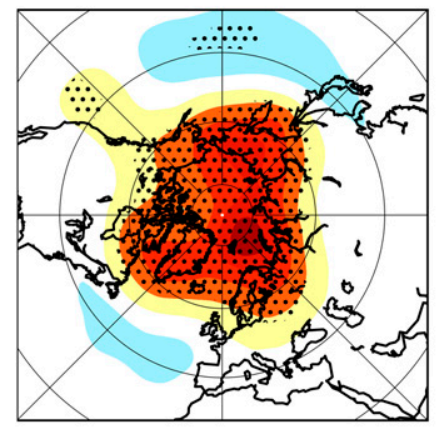

d) T159 1st

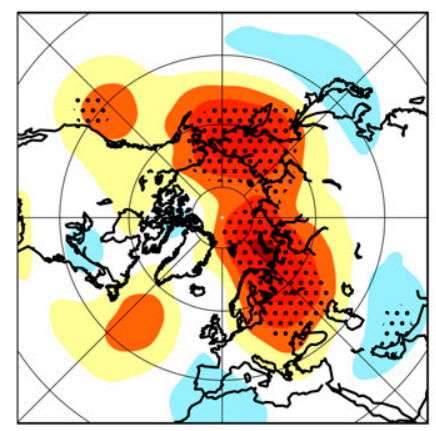

b) T511

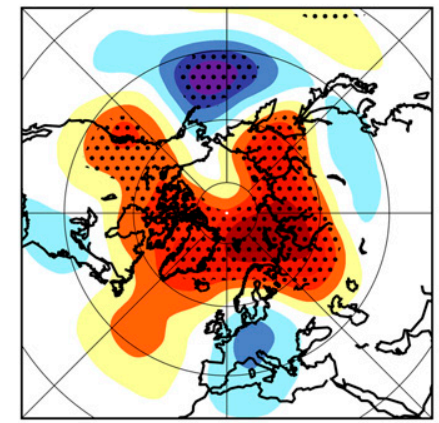

e) T159 2nd

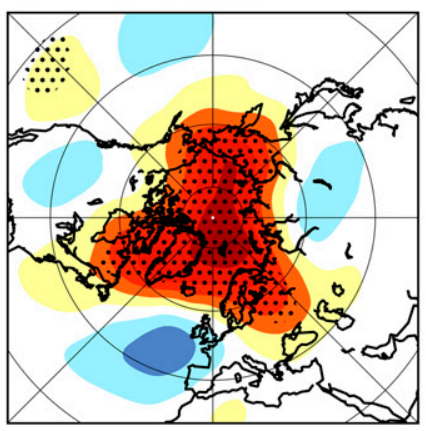

C) $\mathrm{T} 1279$

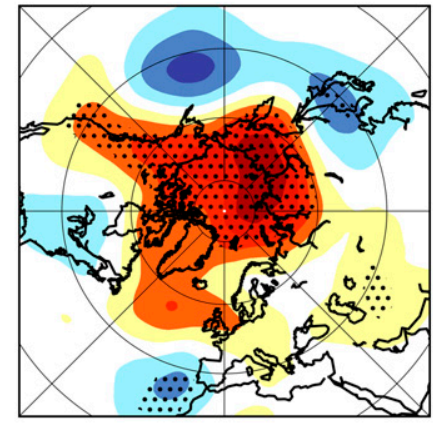

f) $T 1593 \mathrm{rd}$

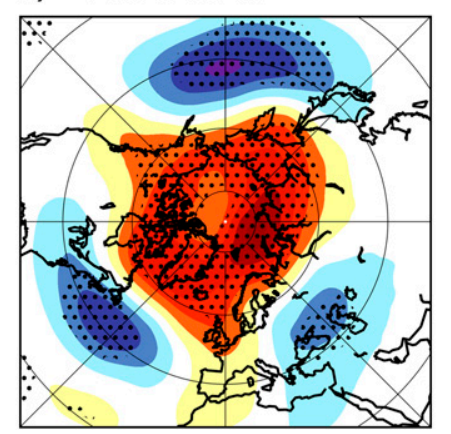

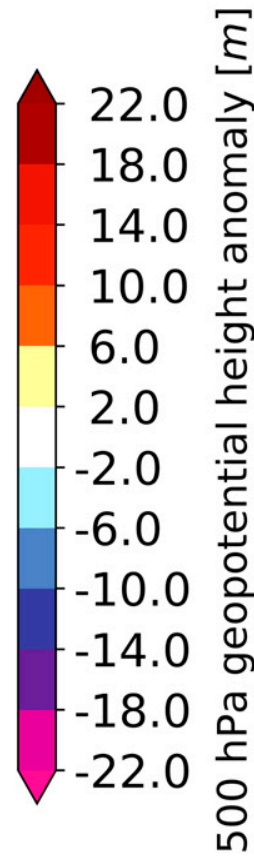

FIG. 5. (top) Difference in mean 500-hPa geopotential height (in $\mathrm{m}$ ) between PAMIP ensembles forced with present day and future Arctic sea ice concentrations for the extended Northern Hemisphere winter season (DJFM) and three different resolutions: (a) $\mathrm{T}_{\mathrm{L}} 159$, (b) $\mathrm{T}_{\mathrm{L}}$ 511, and (c) $\mathrm{T}_{\mathrm{L}}$ 1279. Ensembles had 300, 100, and 100 members, respectively, as described in section 3. (bottom) The 300-member $\mathrm{T}_{\mathrm{L}} 159$ ensemble is displayed for different 100-member chunks separately.

four key variables, the 2-m air temperature (T2M), mean surface level pressure (MSLP), 500-hPa geopotential height (z500), and 500-hPa zonal wind velocity (u500) in the midlatitudes $\left(30^{\circ}-70^{\circ} \mathrm{N}\right)$. 1) For each resolution and variable we calculate the mean over 100 ensemble members of the forcing-induced differences. 2) Next, we apply bootstrapping to draw random ensembles of increasing size, starting from size 1 up to 100 . 3) We then calculate the RMSE between the means of the real and the resampled ensembles. 4) Finally we normalize the results by dividing the RMSE by the field mean of the absolute of the real ensemble mean. Steps 2 through 4 are repeated $B=1000$ times to even out random behavior of the bootstrapped ensembles, especially at low sizes. The resultant specific RMSE delineates the power of an ensemble with a given size to predict the ensemble mean for the chosen variable and resolution. Note that predicting the ensemble mean of a model with said model is not equal to prediction of observations, and we expect the RMSE to decrease according to $1 / \sqrt{n}$ due to the suppression of random noise (Scaife and Smith 2018). Using the high-resolution $T_{L} 1279$ as our base we can calculate the decrease or increase of predictive power for a given ensemble size. Table 1 shows that the number of ensemble members needed to reach the same signal-to-noise ratio as

$$
n_{\mathrm{T}_{\mathrm{L}} 159}=\left(\frac{\sqrt{n_{\mathrm{T}_{\mathrm{L}} 1279}}}{S_{\mathrm{T}_{\mathrm{L}} 159}}\right)^{2} \text {, }
$$

with $n$ being the number of ensemble members, and $S$ the scaling factor for the given resolution and variable. Since ensembles of roughly equal predictive power happen to be of similar size, we argue that the midlatitude response variability observed between the three subsets of the $\mathrm{T}_{\mathrm{L}} 159$ ensemble is a good indicator for the response variability to be expected from 300 ensemble member $\mathrm{T}_{\mathrm{L}} 511$ or $\mathrm{T}_{\mathrm{L}} 1279$ simulations. And while the convergence to the ensemble mean does happen faster at $T_{L} 511$ than at $T_{L} 159$, we argue that this does not constitute an interpretable result, since the highest-resolution ensemble also converges slower than $\mathrm{T}_{\mathrm{L}} 511$ for all the 2-m air temperature.

\section{a. Beyond the local model response}

The zonal average temperature plots in Figs. $3 \mathrm{a}-\mathrm{c}$ illustrate how the polar surface forcing leads to a strong response in the Arctic lower troposphere in all resolutions. With increasing height and distance from the sea ice forcing, the temperature response weakens. This response makes the temperature stratification less stable in the lower troposphere. Furthermore, the meridional temperature gradient is 
a) T159 total

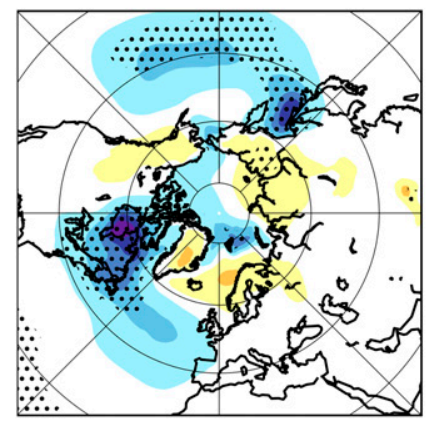

d) T159 1st

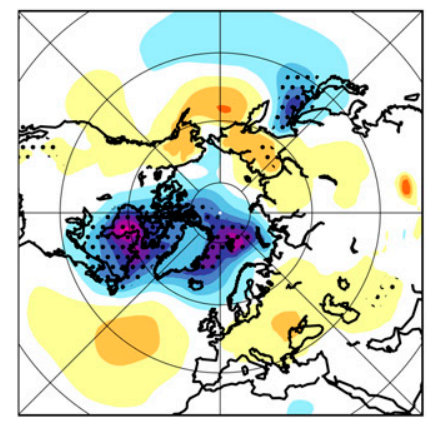

b) $\mathrm{T} 511$

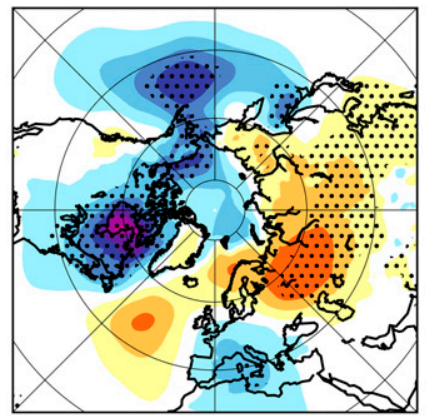

e) T159 2nd

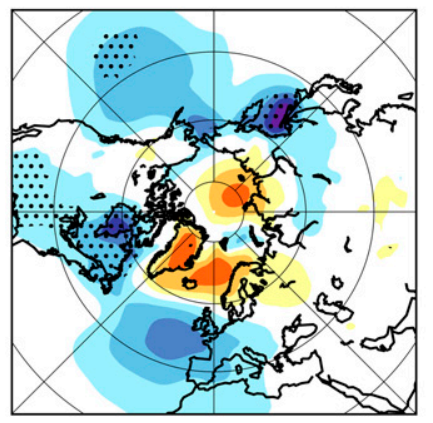

C) $\mathrm{T} 1279$

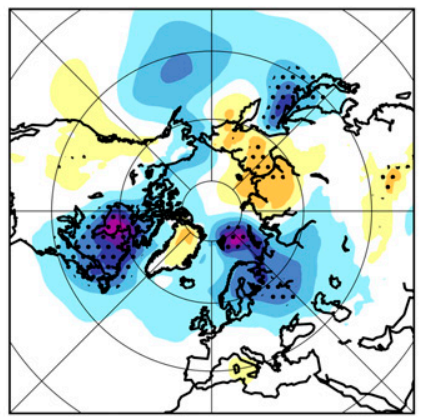

f) $T 1593 \mathrm{rd}$

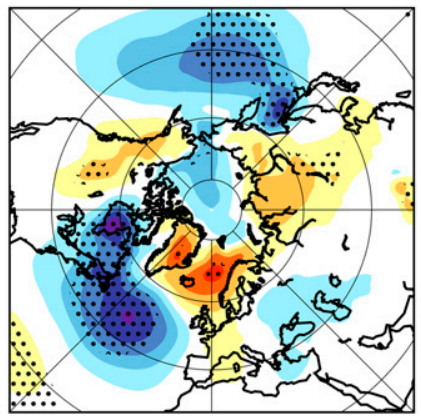

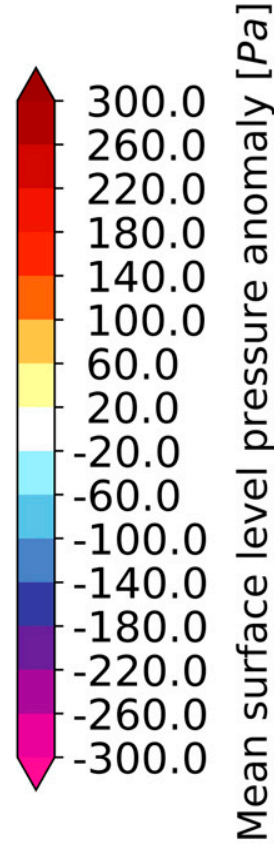

FIG. 6. As in Fig. 5, but for sea level pressure (in Pa).

weakened especially at the surface through the stronger heating at high compared to low latitudes. Figures $3 b$ and $3 c$ were obtained from 100 -ensemble-member simulations while the low-resolution Fig. $3 \mathrm{a}$ is based on 300 ensemble members. The second row of plots (Figs. 3d-f) splits the dataset from Fig. 3a into three ensembles of 100 members, allowing a fair comparison with the higher-resolution simulations and, at the same time, permitting us to understand the remaining AIV at the given ensemble size, especially in the polar stratosphere. The stratospheric response is inconsistent between the different ensembles, independent of the resolution. As shown before, the convergence toward the ensemble mean is very similar across resolutions.

The reduced meridional temperature gradient shown in Fig. 3, through a reduced pressure gradient, results in a weakened polar jet stream, as seen in Figs. $4 \mathrm{a}-\mathrm{c}$. For the $\mathrm{T}_{\mathrm{L}} 159$ and $T_{L} 511$ simulations a slight southward shift of the jet stream can be seen. Once again splitting the 300-member ensemble from Fig. 4a we observe that the internal variability in the $\mathrm{T}_{\mathrm{L}} 159$ results is larger than the difference between resolutions. We obtained Figs. 4d-f from the exact same model setup simply by changing the initial conditions. This shows that even using an ensemble with as many as 100 members can yield very different but still seemingly statistically significant responses, depending on which set of equally valid initial conditions one happens to choose. This can explain contrasting results in previous studies. Here we show that the differences in signal are purely the result of AIV.
In summary, the zonal mean response of temperature and zonal wind to sea ice decline is remarkably similar across a wide range of horizontal resolutions and the differences between resolutions are smaller than those between subsamples at constant resolution. As we will subsequently show, the same is essentially true for spatially resolved patterns as well.

\section{b. A spatial picture}

The heating of the lower troposphere results in lifting of the 500-hPa geopotential height, centered on the forcing region for all three resolutions, shown in Figs. 5a-c. While the spatial pattern is broadly similar, regional differences exist, such as over the Bering Strait and Chukchi Sea, where the $T_{L} 511$ ensemble shows no response. Once again Figs. 5d-f show the lowresolution simulations 300 ensemble members split into three equally sized subsets. The differences between the subsets caution us against interpreting the midlatitude response differences in Figs. 5a-c. Evidently, a strong response over the North Atlantic and Pacific can exist and seems to be even significant in one subset, while another realization exhibits no such response.

Analysis of the mean sea level pressure (MSLP) in Fig. 6 shows that off the Canadian coast the simple baroclinic lifting response seen from negative sea level pressure anomalies dominates. Meanwhile, in the central Arctic and along the Siberian coasts, the model response is mostly insignificant at the surface while there is a significant increase in all ensembles at $500 \mathrm{hPa}$ (Fig. 5). This can be interpreted as baroclinic lifting 


\section{a) T159 total}

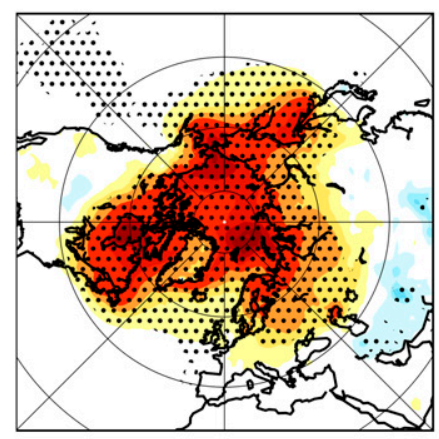

d) T159 1st

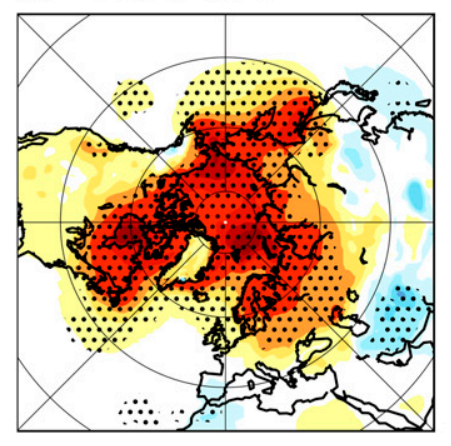

b) $\mathrm{T} 511$

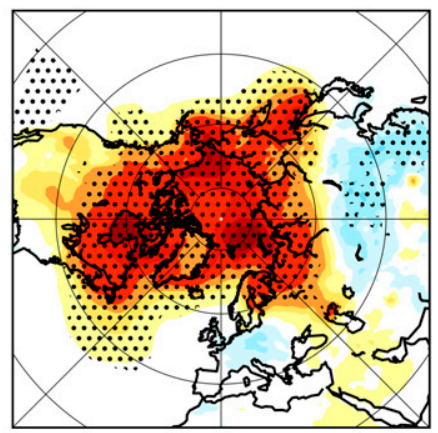

e) T159 2nd

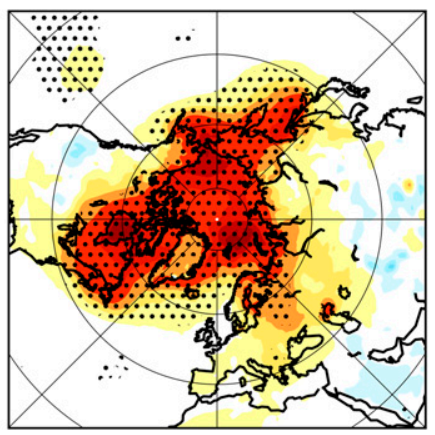

C) $\mathrm{T} 1279$

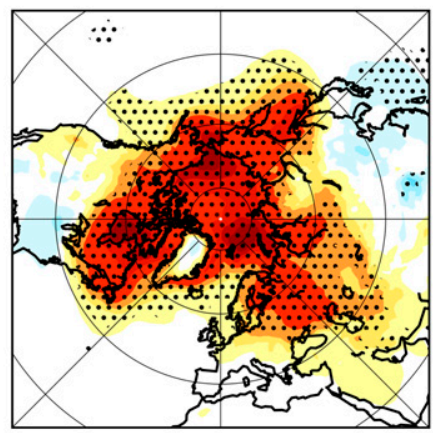

f) $\mathrm{T159} 3 \mathrm{rd}$

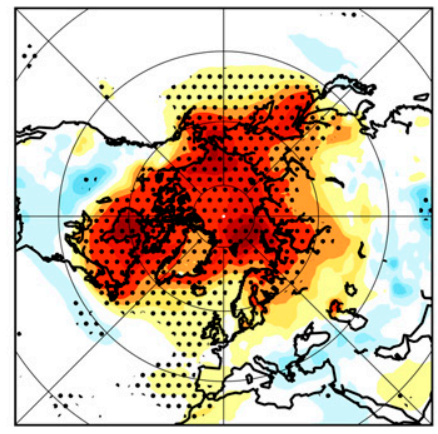

7.0 5.0 $3.0 \unrhd$ $1.0 \frac{\pi}{\pi}$ 0.5 0.3 0.3 0.1 $-0.1$ $-0.3$ $-0.5$ $-1.0$ $-3.0$ $-5.0$ $-7.0$

FIG. 7. As in Fig. 5, but for mean air temperature at 2-m height (in K).

superimposed by a barotropic component of sea level pressure and $500-\mathrm{hPa}$ geopotential increase. We do not see a strong influence of resolution on the existence and strength of this second-order effect. In the midlatitudes mean sea level pressure responses are different from ensemble to ensemble. The internal variability shown in Figs. 6d-f inhibits interpretation. We consider it noteworthy that, had we only performed simulations at $\mathrm{T}_{\mathrm{L}} 159$ and $\mathrm{T}_{\mathrm{L}} 511$, we would have concluded that the higher resolution is required to simulate a sea ice decline induced strengthening of the Siberian high in OpenIFS. A further resolution increase to $\mathrm{T}_{\mathrm{L}} 1279$ does however not replicate this striking result.

The spatial extent of the DJFM near-surface temperature response can be seen in Fig. 7. Areas directly affected by the thermodynamic forcing experience up to a 7-K increase in 2-m air temperature, the effects of which are advected both upward in the atmospheric boundary layer and horizontally into nearby regions. Despite the undersampling of the $1^{\circ}$ forcing field at low resolution and oversampling it at high resolutions, the local near-surface temperature response does not differ widely between different experiments. The impact at a distance from the forcing area is characterized by sizable temperature increases of up to $3 \mathrm{~K}$ south of the Arctic Ocean over the northern continental areas, and no change or cooling of up to $1 \mathrm{~K}$ in selected Asian regions, which are different depending on the ensemble. Over the oceans, the 2-m temperature closely follows the prescribed SST and thus cannot react freely. Southern Europe and the southern areas of North America exhibit no significant T2M response to the sea ice forcing. The differences between the resolutions in Figs. 7a-c are of a similar order of magnitude as those between the $T_{L} 159$ subsamples in Figs. 7d-f.

A frequently discussed potential link is that the weakened polar jet stream may lose positional stability, resulting in a wavier jet and subsequently more frequent blocking and extreme weather events. We first employ the sinuosity metric developed by Cattiaux et al. (2016), whereby the waviness of midlatitude $\left(30^{\circ}-70^{\circ} \mathrm{N}\right)$ flow is computed by dividing the length of the daily $500-\mathrm{hPa}$ height isohypse by the length of $50^{\circ} \mathrm{N}$ parallel. Figure 8 shows that changes to the mean sinuosity are small compared to the ensemble standard deviations, and not uniform in direction. These results are independent of the model resolution. Recent findings by Blackport and Screen (2020a) also conclude that when including the most recent years no trend toward a wavier jet stream can be seen in observational data.

In light of the previous results, we would also expect the midlatitude blocking frequency not to have changed substantially. We employ the 2D blocking metric developed by Davini et al. (2012) and depict our findings in Fig. 9. In the vicinity of the forcing area over Iceland, the Denmark Strait, and parts of southern Greenland and the Norwegian Sea the number of blocking events increased at all resolutions. Only the third subset of $\mathrm{T}_{\mathrm{L}} 159$ ensemble does not show a significant trend here. Increased East Siberian blocking can also be observed. However, the location of significant changes does not overlap 
a) Northern Hemisphere

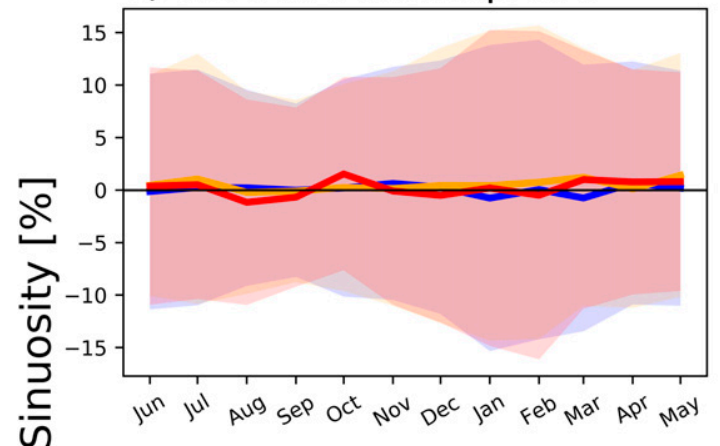

d) Asia

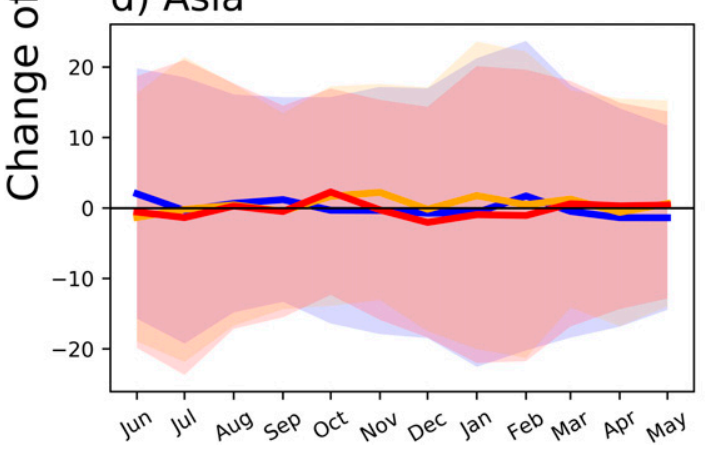

b) Europe

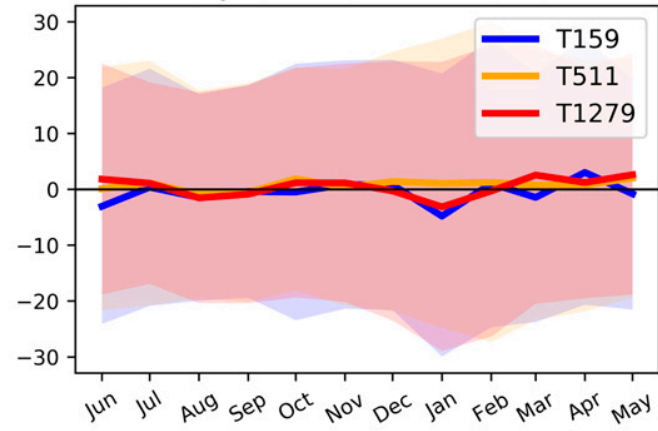

c) North America

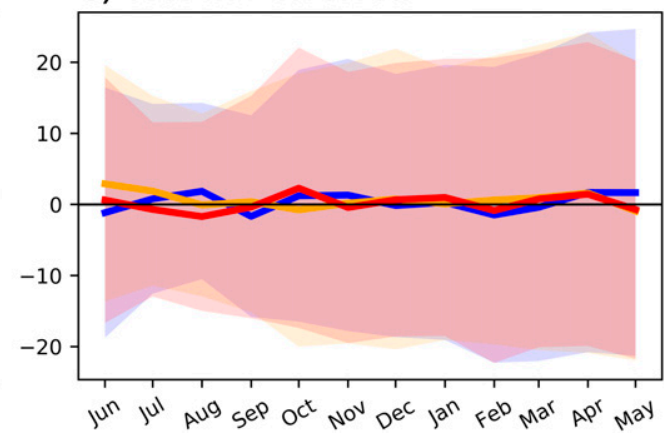

FIG. 8. Percentage-wise differences of the sinuosity metric for future sea ice forcing vs present-day conditions (solid lines), representing the length of the $500-\mathrm{hPa}$ isohypse divided by the length of $50^{\circ} \mathrm{N}$ parallel (Cattiaux et al. 2016). Sinuosity response for (a) the entire Northern Hemisphere, (b) Europe $\left(0^{\circ}-65^{\circ} \mathrm{E}\right)$, (c) Asia $\left(65^{\circ}-130^{\circ} \mathrm{E}\right)$, and (d) North America $\left(60^{\circ}-125^{\circ} \mathrm{W}\right)$. The shaded areas denote the percentage-wise sum of the ensemble standard deviations of the present-day and future forcing simulations.

as consistently as in the Atlantic. Depending on the selection of the $\mathrm{T}_{\mathrm{L}} 159$ subset we could either interpret a resolutiondependent trend of the response with subsets 2 and 3 or see no trend with subset 1 . Therefore AIV evidently inhibits the analysis of the resolution dependence of the blocking response. Away from the vicinity of the sea ice forcing midlatitude blocking events do not seem to be affected in any major way at any model resolution. Furthermore, we find no response of the average duration of blocking events to the sea ice concentration forcing (results not shown).

Finally, we look at the concrete impact of the forcing on extreme cold event frequency in four separate regions, the Arctic $\left(75^{\circ}-90^{\circ} \mathrm{N}\right)$, Europe $\left(30^{\circ}-60^{\circ} \mathrm{N}, 0^{\circ}-65^{\circ} \mathrm{E}\right)$, Asia $\left(30^{\circ}-\right.$ $\left.60^{\circ} \mathrm{N}, 65^{\circ}-130^{\circ} \mathrm{E}\right)$, and North America $\left(30^{\circ}-60^{\circ} \mathrm{N}, 60^{\circ}-125^{\circ} \mathrm{W}\right)$, shown in Fig. 10. We adapt the method described in Zschenderlein et al. (2019), whereby the number of events for which at least $5 \times 10^{6} \mathrm{~km}^{2}$ of the given area have reached the 5 th percentile of low temperatures for at least three days in a row is counted. The reference period for the percentile is a \pm 15 -day moving window in the PD ensemble around each day of the extended winter season (DJFM). Not surprisingly, the future Arctic, directly affected by the heat flux from the ocean surface into the lower atmosphere and with temperatures on average $\sim 4 \mathrm{~K}$ higher than present day, sees many fewer events that are considered cold extremes by current standards. At the midlatitudes North America sees the largest reduction of extreme cold events in our simulations, contrary to sometimes reported intensification of blizzards in the region (Cohen et al. 2018a). In Asia, the forcing has comparatively little effect, while the European sector response lies in between the other two. Crucially, the model resolution does not have a clear impact on the response of extreme cold events to reduced sea ice concentration forcing.

\section{c. Further metrics}

A number of additional metrics were calculated and exhibited either no significant response to the forcing or no difference between the resolutions. These include the polar cap height, the Eliassen-Palm flux, the synoptic eddy vorticity forcing, and the North Atlantic Oscillation. For brevity's sake we omit showing figures of the results. A toolbox to reproduce every metric and a DOI allowing for the download of the underlying dataset are provided in the appendix.

\section{Conclusions}

Our study sought to address the question of whether the conflicting results of recent model-based studies of the wintertime linkages from reduced Arctic SIC to the midlatitudes are partially caused by the comparatively low horizontal resolutions that were employed. Following the PAMIP 
a) T159 total

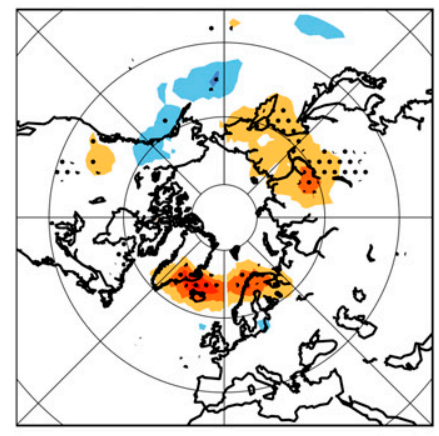

d) T159 1st

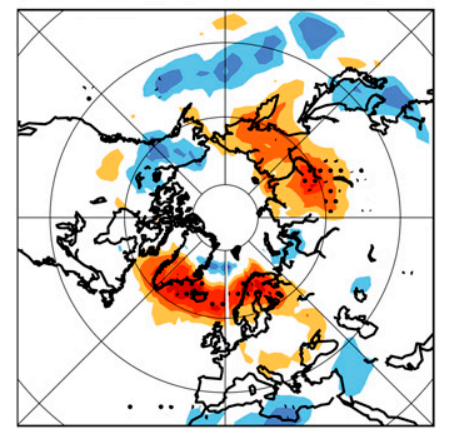

b) T511

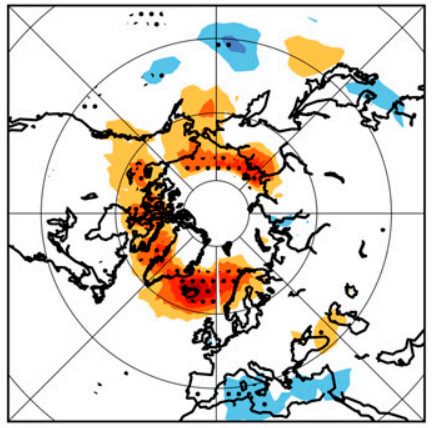

e) T159 2nd

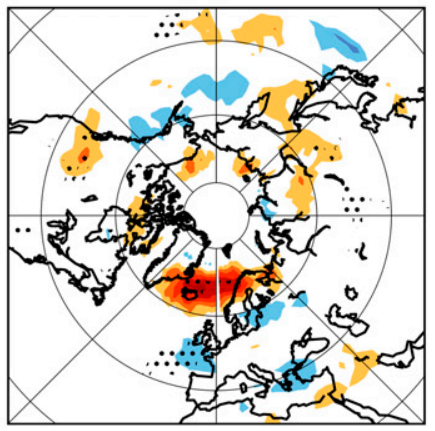

C) $\mathrm{T} 1279$

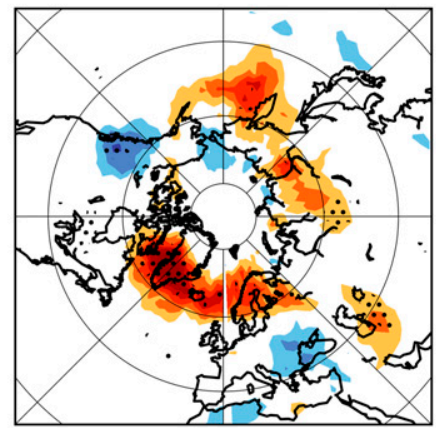

f) $\mathrm{T} 159 \mathrm{3rd}$

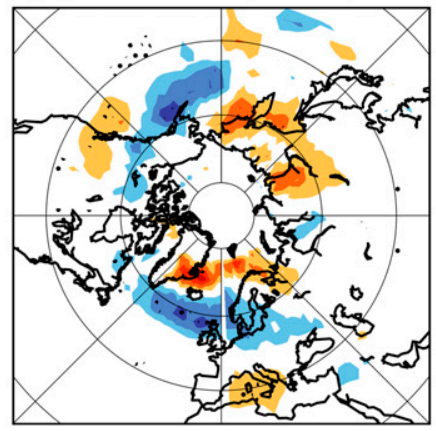

FIG. 9. (top) Change of the Northern Hemisphere blocking index (in \%) for future sea ice forcing relative to present-day conditions for PAMIP experiments at three different resolutions: (a) $T_{L} 159$, (b) $T_{L} 511$, and (c) $T_{L} 1279$. Results are based on the method proposed by Davini et al. (2012). Resolution anomalies of the forcing-induced differences. Dots indicate the $95 \%$ bootstrapping confidence interval. Ensembles consist of 300,100, and 100 members, respectively, as described in section 3. (bottom)The 300-member $T_{L} 159$ ensemble split into equally sized subsets.

experimental design, we did not find any evidence that key midlatitude responses to sea ice decline would be affected by increasing the resolution from $\mathrm{T}_{\mathrm{L}} 159(125 \mathrm{~km}, 91$ layers) first to $\mathrm{T}_{\mathrm{L}} 511(39 \mathrm{~km}, 91$ layers $)$ and finally to $\mathrm{T}_{\mathrm{L}} 1279(16 \mathrm{~km}$, 137 layers). On the one hand, we find that following the minimum ensemble size requirement of 100 members set by PAMIP can seemingly lead to nonrobust statistically significant responses. On the other hand, assuming the model response is realistic, the physical importance of a midlatitude response that is not even robust in a 100-yr sample is limited. The PAMIP protocol nevertheless provides a good basis for high-resolution atmosphere modeling experiments, as individual years can be calculated with applicationlevel parallelization. We acknowledge the limitations of the chosen PAMIP protocol experiments, wherein keeping the SSTs at present-day values, and omitting longer-term ocean dynamical changes, results in the absence of a summertime response.

We conclude that atmospheric horizontal resolution has negligible influence on the outcome of model-based investigation of Arctic SIC decline to midlatitude linkages. Given the inherent cost of high-resolution climate modeling we recommend further research into these links to focus computational expenditure on increased ensemble size or coupled models.
Acknowledgments. The work benefited from funding from the European Union's Horizon 2020 Research and Innovation programme through Grant Agreement 727862 APPLICATE. This is a contribution to the Year of Polar Prediction (YOPP), a flagship activity of the Polar Prediction Project (PPP), initiated by the World Weather Research Programme (WWRP) of the World Meteorological Organization (WMO). We acknowledge the WMO WWRP for its role in coordinating this international research activity. L.Z. acknowledges the financial support by the Federal Ministry of Education and Research of Germany in the framework of SSIP (Grant 01LN1701A). This paper is a contribution to the project L4 of the Collaborative Research Centre TRR 181 "Energy Transfers in Atmosphere and Ocean" funded by the Deutsche Forschungsgemeinschaft (DFG, German Research Foundation) under Project 274762653. We thank the Jülich Supercomputing Centre for providing a share of the JUWELS ESM Partition under the compute projects chhb19, chhb20, and cesmtst as well as the data storage project hhb19. The authors gratefully acknowledge the Gauss Centre for Supercomputing e.V. (www.gauss-centre.eu) for funding this project by providing computing time through the John von Neumann Institute for Computing (NIC) on the GCS Supercomputer JUWELS at Jülich Supercomputing 

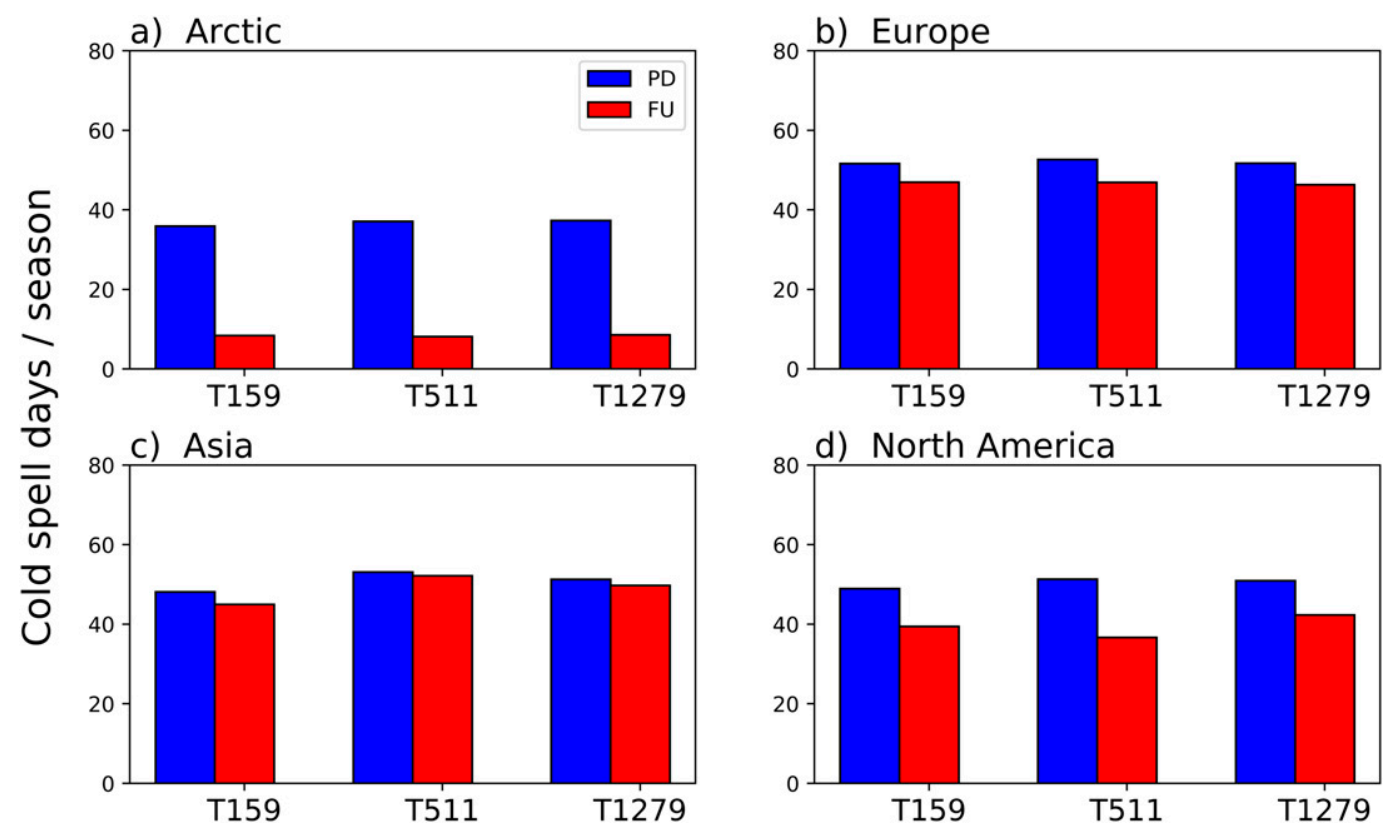

FIG. 10. Change of the number of cold-spell days per extended winter season (DJFM), where a cold spell is defined as a period of at least three consecutive days with at least $500000 \mathrm{~km}^{2}$ of the given area experiencing temperatures less than the 5th percentile of temperatures on the present-day simulation's 31-day window. The areas are defined as (a) Arctic $\left(75^{\circ}-90^{\circ} \mathrm{N}\right)$, (b) Europe $\left(30^{\circ}-60^{\circ} \mathrm{N}, 0^{\circ}-65^{\circ} \mathrm{E}\right)$, (c) Asia $\left(30^{\circ}-60^{\circ} \mathrm{N}, 65^{\circ}-130^{\circ} \mathrm{E}\right)$, and (d) North America $\left(30^{\circ}-60^{\circ} \mathrm{N}, 60^{\circ}-125^{\circ} \mathrm{W}\right)$ The method is adapted from Zschenderlein et al. (2019). Results from ensembles of size 100 are shown.

Centre (JSC). We also thank the Helmholtz ESM-Project for technical support.

\section{APPENDIX}

\section{Toolbox and Dataset}

To encourage reproduction of results and further use of the data we share the toolbox used to generate the results shown, as well underlying dataset. We provide access to the six datasets via the World Data Centre for Climate (Streffing et al. (2021a,b,c,d,e,f). Scripts to recreate the analysis and shown figures can be found at https://github.com/JanStreffing/2019_PAMIP_JCLI_PAPER. Furthermore, these scripts use the Mid-Latitude Evaluation System available from https:/github.com/oloapinivad/MiLES (Davini et al. 2012).

\section{REFERENCES}

Baker, A. J., and Coauthors, 2019: Enhanced climate change response of wintertime North Atlantic circulation, cyclonic activity, and precipitation in a $25-\mathrm{km}$-resolution global atmospheric model. J. Climate, 32, 7763-7781, https://doi.org/10.1175/JCLI-D19-0054.1.

Bauer, P., and T. Richardson, 2014: New model cycle 40r1. ECMWF Newsletter, No. 138, ECMWF, Reading, United Kingdom, p. 3 , https://www.ecmwf.int/node/14581.

Blackport, R., and J. A. Screen, 2020a: Insignificant effect of Arctic amplification on the amplitude of midlatitude atmospheric waves. Sci. Adv., 6, eaay2880, https://doi.org/10.1126/sciadv.aay2880. $\longrightarrow$, and $\longrightarrow, 2020 \mathrm{~b}$ : Weakened evidence for mid-latitude impacts of Arctic warming. Nat. Climate Change, 10, 1065-1066, https://doi.org/10.1038/s41558-020-00954-y.

,,-- K. van der Wiel, and R. Bintanja, 2019: Minimal influence of reduced Arctic sea ice on coincident cold winters in mid-latitudes. Nat. Climate Change, 9, 697-704, https://doi.org/ 10.1038/s41558-019-0551-4.

Cattiaux, J., Y. Peings, D. Saint-Martin, N. Trou-Kechout, and S. J. Vavrus, 2016: Sinuosity of midlatitude atmospheric flow in a warming world. Geophys. Res. Lett., 43, 8259-8268, https:// doi.org/10.1002/2016GL070309.

Chen, H. W., R. B. Alley, and F. Zhang, 2016a: Interannual Arctic sea ice variability and associated winter weather patterns: A regional perspective for 1979-2014. J. Geophys. Res. Atmos., 121, 14433, https://doi.org/10.1002/2016JD024769.

- F. Zhang, and R. B. Alley, 2016b: The robustness of midlatitude weather pattern changes due to Arctic sea ice loss. J. Climate, 29, 7831-7849, https://doi.org/10.1175/JCLI-D-16-0167.1.

Cohen, J., and Coauthors, 2014: Recent Arctic amplification and extreme mid-latitude weather. Nat. Geosci., 7, 627-637, https://doi.org/10.1038/ngeo2234.

— K. Kfeiffer, and J. A. Francis, 2018a: Warm Arctic episodes linked with increased frequency of extreme winter weather in the United States. Nat. Commun., 9, 869, https://doi.org/ 10.1038/s41467-018-02992-9.

- , and Coauthors, 2018b: Arctic change and possible influence on mid-latitude climate and weather: A U.S. CLIVAR White Paper 2018-1. K. Uhlenbrock, Ed., U.S. CLIVAR Project Office, 41 pp., https://doi.org/10.5065/D6TH8KGW.

— , and Coauthors, 2019: Divergent consensuses on Arctic amplification influence on midlatitude severe winter weather. 
Nat. Climate Change, 10, 20-29, https://doi.org/10.1038/ s41558-019-0662-y.

Davini, P., and F. D'Andrea, 2016: Northern Hemisphere atmospheric blocking representation in global climate models: Twenty years of improvements? J. Climate, 29, 8823-8840, https://doi.org/10.1175/JCLI-D-16-0242.1.

— C. Cagnazzo, S. Gualdi, and A. Navarra, 2012: Bidimensional diagnostics, variability, and trends of Northern Hemisphere blocking. J. Climate, 25, 6496-6509, https://doi.org/10.1175/ JCLI-D-12-00032.1.

— , S. Corti, F. D'Andrea, G. Rivière, and J. von Hardenberg, 2017: Improved winter European atmospheric blocking frequencies in high-resolution global climate simulations. J. $A d v$. Model. Earth Syst., 9, 2615-2634, https://doi.org/10.1002/ 2017MS001082.

ECMWF, 2014a: IFS documentation. Part III: Dynamics and numerical procedures. IFS documentation, CY40R1, ECMWF, 29 pp., https://www.ecmwf.int/sites/default/files/elibrary/2014/ 9203-part-iii-dynamics-and-numerical-procedures.pdf.

— 2014b: IFS documentation. Part IV: Physical processes. IFS documentation, CY40R1, ECMWF, 190 pp., https:// www.ecmwf.int/sites/default/files/elibrary/2014/9204-part-iv-physical-processes.pdf.

— 2014c: IFS documentation. Part VII: ECMWF wave model. IFS documentation, CY40R1, ECMWF, 79 pp., https:// www.ecmwf.int/sites/default/files/elibrary/2014/9207-part-viiecmwf-wave-model.pdf.

Efron, B., and R. Tibshirani, 1986: Bootstrap methods for standard errors, confidence intervals, and other measures of statistical accuracy. Stat. Sci., 1, 54-75, https://doi.org/10.1214/ss/1177013815.

Forbes, R., A. Geer, K. Lonitz, and M. Ahlgrimm, 2016: Reducing systematic errors in cold-air outbreaks. ECMWF Newsletter, No. 146, ECMWF, Reading, United Kingdom, 17-22, https:// www.ecmwf.int/node/15041.

Haiden, T., and Coauthors, 2015: Evaluation of ECMWF forecasts, including 2014-2015 upgrades. European Centre for MediumRange Weather Forecasts Tech. Memo. 756, 51 pp., https:// doi.org/10.21957/tcsvl3pc.

Hertwig, E., J.-S. von Storch, D. Handorf, K. Dethloff, I. Fast, and T. Krismer, 2015: Effect of horizontal resolution on ECHAM6AMIP performance. Climate Dyn., 45, 185-211, https://doi.org/ 10.1007/s00382-014-2396-x.

Holton, J. R., 2004: An Introduction to Dynamic Meteorology. 4th ed. Academic Press, 535 pp., http://books.google.com/books? id $=$ fhW5oDv3EPsC.

Iles, C. E., R. Vautard, J. Strachan, S. Joussaume, B. R. Eggen, and C. D. Hewitt, 2019: The benefits of increasing resolution in global and regional climate simulations for European climate extremes. Geosci. Model Dev., 13, 5583-5607, https://doi.org/ 10.5194/gmd-2019-253.

Jung, T., and Coauthors, 2012: High-resolution global climate simulations with the ECMWF model in Project Athena: Experimental design, model climate, and seasonal forecast skill. J. Climate, 25, 3155-3172, https://doi.org/10.1175/JCLI-D-11-00265.1.

Kug, J.-S., J.-H. Jeong, Y.-S. Jang, B.-M. Kim, C. K. Folland, S.-K. Min, and S.-W. Son, 2015: Two distinct influences of Arctic warming on cold winters over North America and East Asia. Nat. Geosci., 8, 759-762, https://doi.org/10.1038/ngeo2517.

Luo, D., Y. Xiao, Y. Yao, A. Dai, I. Simmonds, and C. L. Franzke, 2016: Impact of Ural blocking on winter warm Arctic-cold Eurasian anomalies. Part I: Blocking-induced amplification. J. Climate, 29, 3925-3947, https://doi.org/10.1175/JCLI-D-150611.1.
MacKinnon, J. G., 2009: Bootstrap hypothesis testing. Handbook of Computational Econometrics, John Wiley and Sons, 183213, https://doi.org/10.1002/9780470748916.ch6.

McKenna, C. M., T. J. Bracegirdle, E. F. Shuckburgh, P. H. Haynes, and M. M. Joshi, 2018: Arctic sea ice loss in different regions leads to contrasting Northern Hemisphere impacts. Geophys. Res. Lett., 45, 945-954, https://doi.org/10.1002/2017GL076433.

Meleshko, V. P., O. M. Johannessen, A. V. Baidin, T. V. Pavlova, and V. A. Govorkova, 2016: Arctic amplification: Does it impact the polar jet stream? Tellus, 68A, 32330, https://doi.org/10.3402/ tellusa.v68.32330.

Mori, M., Y. Kosaka, M. Watanabe, H. Nakamura, and M. Kimoto, 2019: A reconciled estimate of the influence of Arctic sea-ice loss on recent Eurasian cooling. Nat. Climate Change, 9, 123129, https://doi.org/10.1038/s41558-018-0379-3.

Nakamura, T., K. Yamazaki, K. Iwamoto, M. Honda, Y. Miyoshi, Y. Ogawa, Y. Tomikawa, and J. Ukita, 2016: The stratospheric pathway for Arctic impacts on midlatitude climate. Geophys. Res. Lett., 43, 3494-3501, https://doi.org/10.1002/2016GL068330.

Overland, J. E., K. R. Wood, and M. Wang, 2011: Warm Arctic-cold continents: Climate impacts of the newly open Arctic sea. Polar Res., 30, 15787, https://doi.org/10.3402/polar.v30i0.15787.

— weather to the changing Arctic. Nat. Climate Change, 6, 992999, https://doi.org/10.1038/nclimate3121.

Pithan, F., T. G. Shepherd, G. Zappa, and I. Sandu, 2016: Climate model biases in jet streams, blocking and storm tracks resulting from missing orographic drag. Geophys. Res. Lett., 43, 7231-7240, https://doi.org/10.1002/2016GL069551.

Ritchie, H., C. Temperton, A. Simmons, M. Hortal, T. Davies, D. Dent, and M. Hamrud, 1995: Implementation of the semiLagrangian method in a high-resolution version of the ECMWF forecast model. Mon. Wea. Rev., 123, 489-514, https://doi.org/ 10.1175/1520-0493(1995)123<0489:IOTSLM>2.0.CO;2.

Roberts, C. D., R. Senan, F. Molteni, S. Boussetta, M. Mayer, and S. Keeley, 2018: Climate model configurations of the ECMWF Integrated Forecast System (ECMWF-IFS cycle 43r1) for HighResMIP. Geosci. Model Dev., 11, 3681-3712, https:// doi.org/10.5194/gmd-11-3681-2018.

Scaife, A. A., and D. Smith, 2018: A signal-to-noise paradox in climate science. npj Climate Atmos. Sci., 1, 28, https://doi.org/ 10.1038/s41612-018-0038-4.

— improve seasonal climate predictions? Atmos. Sci. Lett., 20, e922, https://doi.org/10.1002/asl.922.

Schiemann, R., and Coauthor, 2017: The resolution sensitivity of Northern Hemisphere blocking in four $25-\mathrm{km}$ atmospheric global circulation models. J. Climate, 30, 337-358, https:// doi.org/10.1175/JCLI-D-16-0100.1.

Screen, J. A., C. Deser, and L. Sun, 2015: Projected changes in regional climate extremes arising from Arctic sea ice loss. Environ. Res. Lett., 10, 084006, https://doi.org/10.1088/17489326/10/8/084006.

Shaw, T. A., and Coauthors, 2016: Storm track processes and the opposing influences of climate change. Nat. Geosci., 9, 656664, https://doi.org/10.1038/ngeo2783.

Smith, D. M., and Coauthors, 2019: The Polar Amplification Model Intercomparison Project (PAMIP) contribution to CMIP6: Investigating the causes and consequences of polar amplification. Geosci. Model Dev., 12, 1139-1164, https://doi.org/ 10.5194/gmd-12-1139-2019.

Staniforth, A., and J. Côté, 1990: Semi-Lagrangian integration schemes for atmospheric models-A review. Mon. Wea. Rev., 
119, 2206-2223, https://doi.org/10.1175/1520-0493(1991) $119<2206$ :SLISFA $>2.0$. CO 2 .

Stevens, B., and Coauthors, 2019: DYamond: The Dynamics of the Atmospheric general circulation Modeled On Non-hydrostatic Domains. Prog. Earth Planet. Sci., 6, 61, https://doi.org/10.1186/ s40645-019-0304-z.

Streffing, J., T. Semmler, L. Zampieri, and T. Jung, 2021a: OpenIFS 40r1 TL1279L137 PAMIP future $2^{\circ}$ warm world (exp16) AWI. World Data Center for Climate (WDCC) at DKRZ, accessed 7 July 2021, http://cera-www.dkrz.de/WDCC/ui/Compact.jsp? acronym=DKRZ_LTA_995_ds00004.

— — — — — , and —, 2021b: OpenIFS 40r1 TL1279L137 PAMIP present day (exp11). World Data Center for Climate (WDCC) at DKRZ, accessed 7 July 2021, http://cerawww.dkrz.de/WDCC/ui/Compact.jsp?acronym=DKRZ_LTA_ 995_ds00003.

,$--\longrightarrow$, and,- 2021c: OpenIFS 40r1 TL159L91 PAMIP future $2^{\circ}$ warm world (exp16) AWI. World Data Center for Climate (WDCC) at DKRZ, accessed 7 July 2021, http://cera-www.dkrz.de/WDCC/ui/Compact.jsp?acronym = DKRZ_LTA_995_ds00006.

,,--- , and $\longrightarrow$, 2021d: OpenIFS 40r1 TL159L91 PAMIP present day (exp11) AWI. World Data Center for Climate (WDCC) at DKRZ, accessed 7 July 2021, http:// cera-www.dkrz.de/WDCC/ui/Compact.jsp?acronym =DKRZ_ LTA_995_ds00005.

$\ldots,-\ldots$, and $-\frac{-}{-}$, 2021e: OpenIFS 40r1 TL511L91 PAMIP future $2^{\circ}$ warm world (exp16) AWI. World Data
Center for Climate (WDCC) at DKRZ, accessed 7 July 2021, http://cera-www.dkrz.de/WDCC/ui/Compact.jsp?acronym = DKRZ_LTA_995_ds00008.

,,-- , and,- 2021f: OpenIFS 40r1 TL511L91 PAMIP present day (exp11) AWI. World Data Center for Climate (WDCC) at DKRZ, accessed 7 July 2021, http:// cera-www.dkrz.de/WDCC/ui/Compact.jsp?acronym $=$ DKRZ LTA_995_ds00007.

Sun, L., J. Perlwitz, and M. Hoerling, 2016: What caused the recent "warm Arctic, cold continents" trend pattern in winter temperatures? Geophys. Res. Lett., 43, 5345-5352, https://doi.org/ 10.1002/2016GL069024.

van Haren, R., R. J. Haarsma, G. J. Van Oldenborgh, and W. Hazeleger, 2015: Resolution dependence of European precipitation in a state-of-the-art atmospheric general circulation model. J. Climate, 28, 5134-5149, https://doi.org/ 10.1175/JCLI-D-14-00279.1.

Vihma, T., 2014: Effects of Arctic sea ice decline on weather and climate: A review. Surv. Geophys., 35, 1175-1214, https:// doi.org/10.1007/s10712-014-9284-0.

Willison, J., W. A. Robinson, and G. M. Lackmann, 2015: North Atlantic storm-track sensitivity to warming increases with model resolution. J. Climate, 28, 4513-4524, https://doi.org/ 10.1175/JCLI-D-14-00715.1.

Zschenderlein, P., A. H. Fink, S. Pfahl, and H. Wernli, 2019: Processes determining heat waves across different European climates. Quart. J. Roy. Meteor. Soc., 145, 2973-2989, https:// doi.org/10.1002/qj.3599. 